\title{
A Novel Coupled Resonator Photonic Crystal Design in Lithium Niobate for Electrooptic Applications
}

\author{
Birol Ozturk, ${ }^{1,2}$ Ozgur Yavuzcetin, ${ }^{1,3}$ and Srinivas Sridhar ${ }^{1}$ \\ ${ }^{1}$ Electronic Materials Research Institute and Department of Physics, Northeastern University, Boston, MA 02115, USA \\ ${ }^{2}$ Department of Physics and Engineering Physics, Morgan State University, Baltimore, MD 21251, USA \\ ${ }^{3}$ Department of Physics, University of Wisconsin-Whitewater, Whitewater, WI 53190, USA \\ Correspondence should be addressed to Srinivas Sridhar; s.sridhar@neu.edu
}

Received 5 June 2015; Accepted 11 October 2015

Academic Editor: Samir K. Mondal

Copyright (C) 2015 Birol Ozturk et al. This is an open access article distributed under the Creative Commons Attribution License, which permits unrestricted use, distribution, and reproduction in any medium, provided the original work is properly cited.

\begin{abstract}
High-aspect-ratio photonic crystal air-hole fabrication on bulk Lithium Niobate (LN) substrates is extremely difficult due to its inherent resistance to etching, resulting in conical structures and high insertion losses. Here, we propose a novel coupled resonator photonic crystal (CRPC) design, combining a coupled resonator approach with that of Bragg gratings. CRPC design parameters were optimized by analytical calculations and FDTD simulations. CRPC structures with optimized parameters were fabricated and electrooptically tested on bulk LN annealed proton exchange waveguides. Low insertion loss and large electrooptic effect were observed with the fabricated devices, making the CRPC design a promising structure for electrooptic device applications.
\end{abstract}

\section{Introduction}

State-of-the-art low loss electrooptic modulators and electric field sensors are based on Mach-Zehnder interferometers (MZI) and are inherently several centimeters long. Slow light structures such as nanostructured photonic crystals (PCs) with an order of magnitude smaller footprint are needed to increase the interaction of light with the material and amplify the electrooptic effect $[1,2]$. Lithium Niobate (LN) is extensively used in device applications due to its unique electrooptic, piezoelectric, and pyroelectric properties [3-5]. Fabrication of photonic crystal structures on LN has been challenging due to its resistance to etching [6-8]. As a direct patterning method, focused ion beam (FIB) is commonly utilized in photonic crystal fabrication [9]. In this process, the resputtering effect in LN prevents the fabrication of highaspect-ratio air holes, resulting in finite depth holes with conical cross sections (Figure 1(b)). Photonic crystals with these hole geometries were shown to deflect the field into the bulk substrate, causing high insertion losses (Figure 1(d)) [10].

Previously, we fabricated Bragg grating type 1D PCs on bulk LN by FIB milling (Figure 1(a)) [6]. The conical cross sections of the air trenches caused high insertion losses (Figure 1(c)), preventing the use of these structures in practical device applications despite their simulated sharp bandgap edge characteristics.

Here, we report on the design, fabrication, and electrooptical characterization of a novel coupled resonator (CR) photonic crystal design in $\mathrm{LN}$ with a low insertion loss. The new design is comprised of periodic air trenches which are key elements in the fabrication of 1D PC structures such as Bragg gratings and Fabry-Perot resonators [11-13]. Air trenches can be milled deeper compared to air holes due to their large cross sections in the horizontal plane and can achieve reduced conicity compared to circular holes. Furthermore, the air trenches are arranged in a coupled resonator pattern that increases the local optical intensity and minimizes outof-plane scattering. The new CR design, which is a novel variation of the classic CR structure [14], has a small footprint with an active area of 110-micron length and 10-micron width.

In the optical measurements of the fabricated structures, the insertion loss dropped from $50 \mathrm{~dB}$ in Bragg grating devices to $10 \mathrm{~dB}$ in the CRPC devices. The DC electrooptic response was measured as $1 \mathrm{~nm} / \mathrm{V}$ shift in the transmission spectrum. The small footprint, low insertion loss, and 


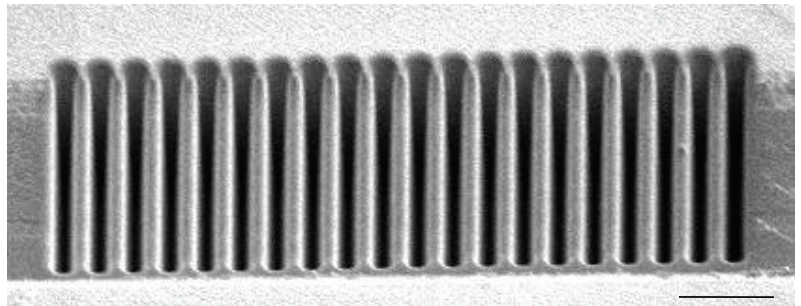

(a)

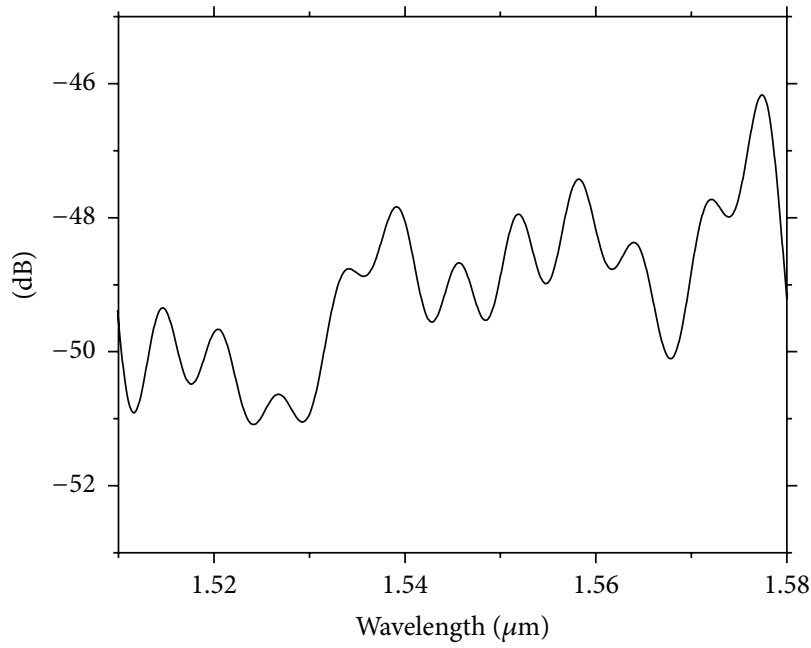

(c)

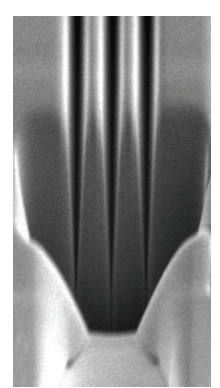

(b)

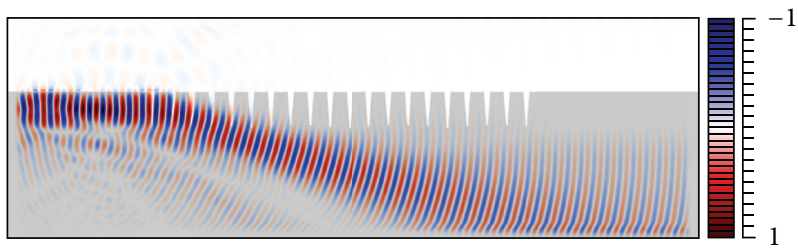

(d)

FIGURE 1: $52^{\circ}$ tilted SEM image of (a) FIB milled Bragg grating on bulk LN (the scale bar denotes $5 \mu \mathrm{m}$ ) and (b) the trenches with conical cross sections. (c) Transmission spectrum of the structure in panel (a) shows high insertion loss up to $51 \mathrm{~dB}$. (d) XZ-plane (side view) FDTD field simulation showing transmission losses due to vertical plane scattering arising from conicity and poor confinement.

electrooptic properties of this new design suggest that CRPC is a promising structure for electrooptic device fabrication.

\section{Materials and Methods}

2.1. Design and Optimization. Figure 2(a) depicts the topview SEM image of FIB milled coupled resonator photonic crystal on an annealed proton exchange (APE) waveguide of a bulk LN crystal [15]. The top and bottom light gray partsdenote the coplanarelectrodes deposited by photolithography prior to FIB milling. The design parameters and the desired high-aspect-ratiocross section of the structure are shown on a cartoon in Figure 2(b). We determined the period of the air trenches analytically using the Bragg Grating Theory. In order to obtain a stop band in the C-band window, we used the third-order grating period $\Lambda=1080 \mathrm{~nm}$ which allows the fabrication of wider air trenches in the direction of field propagation. The other design parameters such as number, width, and length of trenches were optimized using two-dimensional Finite Difference Time Domain (FDTD) simulations that are performed with the freely available MEEP software [16]. The optimized CR photonic crystal had 100 air trenches on each side of the waveguide with $4.5 \mu \mathrm{m}$ and $2.5 \mu \mathrm{m}\left(l_{1}\right.$ and $\left.l_{2}\right)$ alternating gaps. The trenches were $w=250 \mathrm{~nm}$ wide. The central gaps formed a continuous partial APE waveguide which reduces the transmission loss while the side air trenches couple to the transmitted field.

2.2. Fabrication. CR photonic crystals were fabricated on the annealed proton exchange (APE) waveguides of bulk LN substrates which were provided by SRICO Inc. Lithium Niobate wafers were $X$-cut and APE waveguides were fabricated along the $Y$-cut orientation. The end facets of the waveguides were polished and had angle cuts to reduce reflections in the optical measurements.

A Helios NanoLab DualBeam focused ion beam (FIB) system was used to directly mill the CR photonic crystal patterns on the waveguides. This equipment uses $\mathrm{Ga}^{+}$ions and the focused beam diameter changes from a few nanometers up to a few microns depending on the beam current that varies from a few $\mathrm{pA}$ up to $40 \mathrm{nA}$. For optimum milling time and gap width of the trenches, we have used a beam current of $2.8 \mathrm{nA}$ and exposure dose of $150 \mathrm{fC}$. This current and dose resulted in trench cuts with $250 \mathrm{~nm}$ widths. We have designed the patterns using DesignCAD LT and milled them with NPGSTM software. In order to achieve better uniformity, we have used the built-in repeat function while sweeping the beam to mill the trenches. 


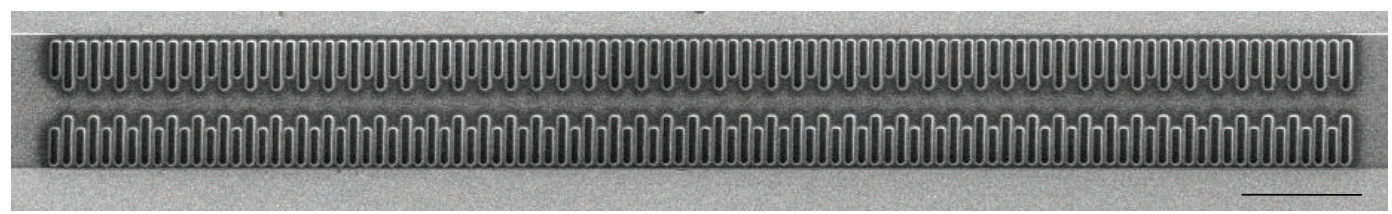

(a)

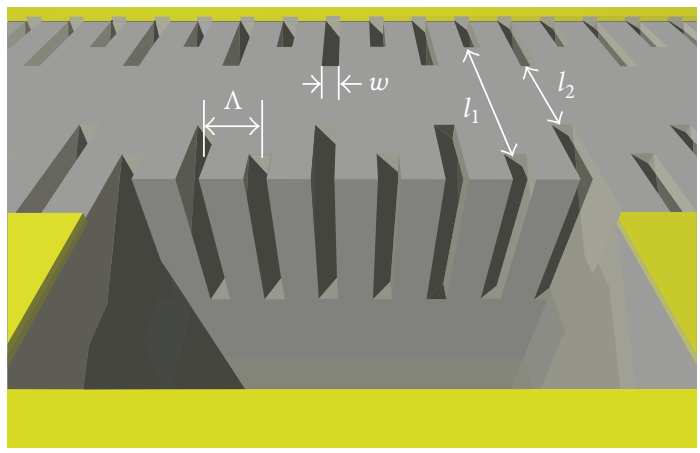

(b)

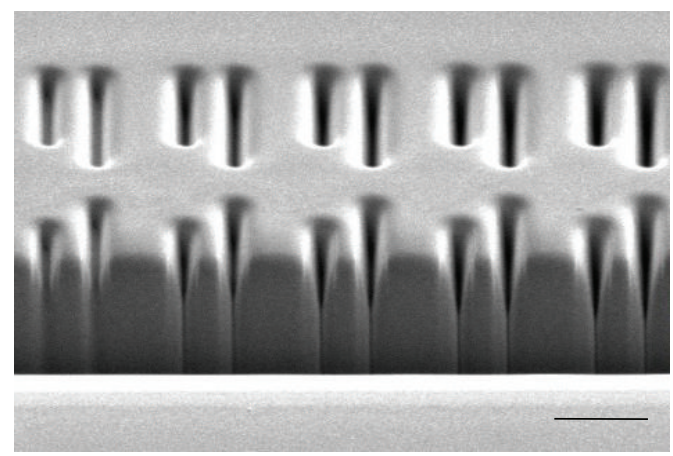

(c)

Figure 2: (a) SEM top-view image of a coupled resonator photonic crystal. Scale bar denotes $10 \mu \mathrm{m}$. (b) A cartoon showing the design parameters of the structure. The cross section shows the expected vertical walls. (c) Tilted SEM image of the FIB milled CR structure. The cross section shows angled side walls and limited depth of air trenches. The scale bar denotes $5 \mu \mathrm{m}$.

FIB patterning was done directly on the APE WGs which can support transverse electric (TE) modes. The widths of the WGs varied from 5 to 7 microns to ensure single-mode transmission for the C-band laser source. Aluminum or gold coplanar electrodes were deposited on each side of the WGs by photolithography for electrooptic measurements. The electrode separation was $10 \mu \mathrm{m}$ and these electrodes were also utilized as guides in the alignment of the CR pattern on the APE WG during the FIB milling. Tilt correction helped the alignment of the WG in the horizontal direction and eliminated the need for alignment marks. Au-Pd was sputter coated on the substrates in order to avoid charging. This coating is needed to select the region of interest with SEM imaging. However, waveguides were not visible after switching to ion beam imaging due to this coating. Thus, low dose ion bombardment of the region was required to remove the Au-Pd coating in order to make the WGs visible prior to CR photonic crystal patterning.

Since FIB milling is a kinetic mechanism, the trench depths were limited due to resputtering effects because it is harder to remove the material from the bottom of the trench at increasing depths which leads to angled side walls on the milled patterns (Figure 1(c)). These angled side walls cause significant deviation in the optical transmission profile of the CR photonic crystals compared to simulated transmission of the initial design with vertical walls as will be discussed consequently.

2.3. FDTD Simulations. Analytically determined CRPC parameters were used in the 2D FDTD simulations to determine the calculated transmitted spectrum with the MEEP software (Figure 3(a), black line with open squares). The spectrum shows a $10 \mathrm{~dB}$ base loss and a dip around $1.55 \mu \mathrm{m}$ in the transmitted signal. The quality factor of this transmission dip was calculated as 166 . Further simulations showed that, for a given set of trench and alternating gap widths, increasing the period of the air trenches red shifts the stop bands (data not shown here). In order to analyze the field-CR photonic crystal interaction, we also performed field pattern simulations. As depicted in Figure 3(b), the field is transmitted through the CR PC while interacting with the structure at a high transmission wavelength $(1.6 \mu \mathrm{m})$ of the spectrum. Figure $3(\mathrm{c})$ shows the field pattern at $1.54 \mu \mathrm{m}$ where the dip occurs. Most of the field is reflected back, similar to Bragg grating, and no transmission is visible at the output of the structure.

2.4. Optical Transmission and Electrooptic Measurements. Optical transmission spectrum was measured via buttcoupling of polarization maintaining single-mode fibers on both ends. The polished end facets of the fibers were cut at an $8^{\circ}$ angle and index matching fluid was used between the fiber tips and the WG facets in order to reduce reflection. An unpolarized Santec TSL-210H C-band tunable fiber laser source was employed for the transmission spectrum measurements in the 1510-1580 $\mathrm{nm}$ range. Fiber laser output was connected to Fiberpro PS-155-A-B polarization scrambler to effectively randomize polarization states. An inline fiber polarizer was used to linearly polarize the laser source before coupling into the APE waveguide. The cartoon in Figure 3(a) inset depicts the fiber coupling of laser source to the APE WG with the CR photonic crystal and electrodes. The transmitted spectrum was collected via another polarization maintaining fiber at the end facet of the waveguide which was coupled to an InGaAs photodetector. Both input and output fibers were aligned to the end facets of the waveguide using Thorlabs Nanomax six axes stages.

The blue line with inverse triangles in Figure 3(a) represents the measured transmission spectrum of the CR 


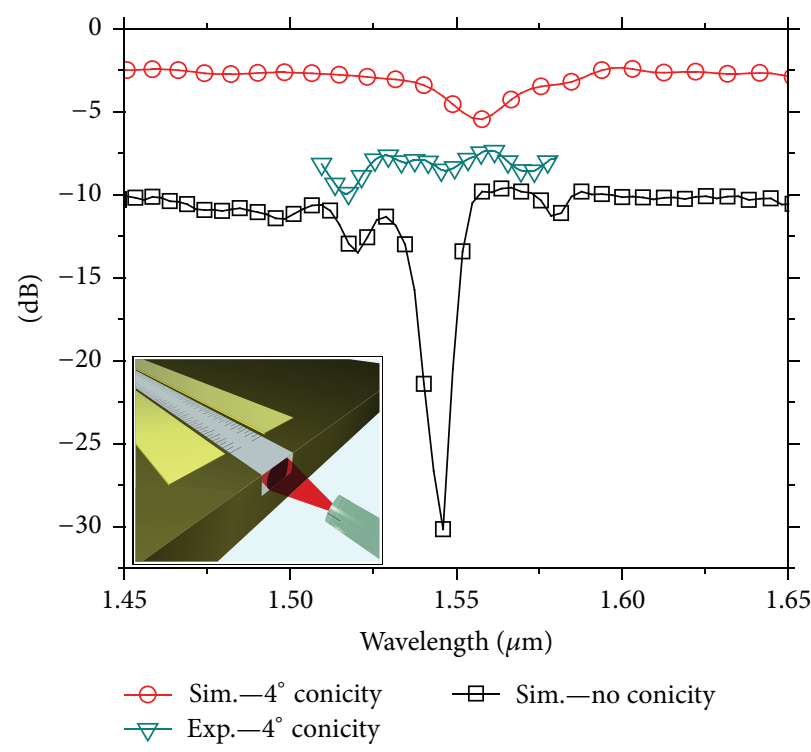

(a)

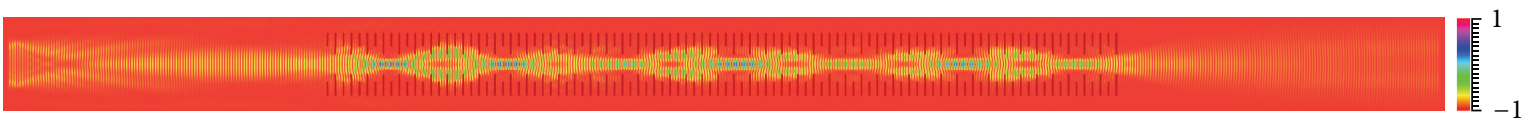

(b)

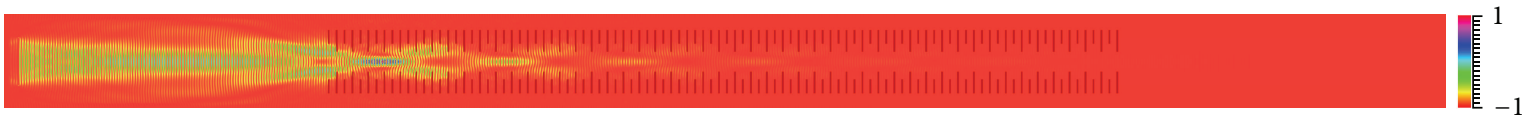

(c)

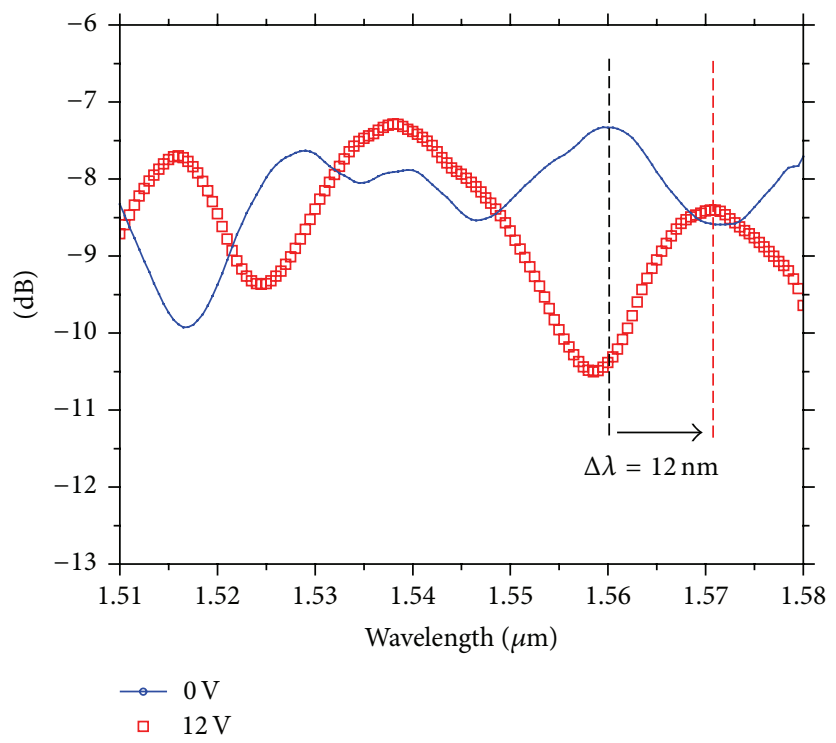

(d)

Figure 3: (a) Measured optical transmission spectrum. Simulated field transmission patterns (b) at $1.6 \mu \mathrm{m}$ and (c) at $1.54 \mu \mathrm{m}$ wavelengths. (d) The electrooptic shift in the optical transmission spectrum.

photonic crystal. There is a large discrepancy between the measured and the expected spectrum (black line) from the simulations: the base transmission loss of the measured spectrum is few $\mathrm{dB}$ less than the expected spectrum; however, a high extinction ratio bandgap edge is not present in the measured spectrum. This discrepancy was previously attributed to the angled wall cross sections (conicity) of the trenches [4]. The depths of the $250 \mathrm{~nm}$ wide trenches were $2 \mu \mathrm{m}$ and the wall angle was $4^{\circ}$ as determined from the SEM cross section images (Figure 2(c)). 3D FDTD simulations 
were carried out to determine the expected transmission spectrum of a CR photonic crystal with the same parameters of the fabricated CR photonic crystal where the depth of the trenches was $2 \mu \mathrm{m}$ with $4^{\circ}$ wall angles. The 3D FDTD simulation result is shown with the red line with open dots in Figure 3(a). The expected transmission loss in this case is lesser but the photonic bandgap does not exist due to conicity. A dip is apparent in the simulation result that does not fit well with the measured spectrum. However, the extinction ratio of this dip is comparable to the extinction ratio of experimentally measured features.

Although a high extinction ratio photonic band edge was not achieved, the low insertion loss $(<10 \mathrm{~dB})$ of the CR photonic crystal makes this new design suitable for device applications. In order to probe the potential use of this novel CR photonic crystal as an electric field sensor, we performed electrooptic measurements.

A Keithley 2400 sourcemeter was employed to apply DC bias to the prefabricated lithographic electrodes to test the electrooptic response of the CR photonic crystal. The red curve with open squares in Figure 3(d) represents the transmission spectrum under positive 10 -volt DC bias. A red shift of $12 \mathrm{~nm}$ was observed in the transmission spectrum with the applied DC voltage corresponding to $1.2 \mathrm{~nm} / \mathrm{V}$ shift in the $10 \mu \mathrm{m}$ electrode separation. The intensity of peaks was also altered with the applied DC voltage where the original spectrum was restored after the DC voltage was turned off. Similar electrooptic shift values were obtained with different samples.

\section{Conclusions}

A novel CR photonic crystal design was fabricated and optically tested. FIB milling was used to directly pattern the CR photonic crystal onto APE waveguides of bulk LN substrates. The measured transmission spectrum of the fabricated CR structures did not display sharp features as the initially simulated design which was attributed to the angled side wall cross sections (conicity) of the air trenches. 3D FDTD simulations of CR designs with angled wall air trenches were in good agreement with the experimental findings. Electrooptical measurements showed significant shift with the applied DC voltage.

While microscale thin film Lithium Niobate devices have been fabricated which can achieve efficient light confinement $[17,18]$, coupling to these devices is quite challenging and typically leads to high insertion losses. By minimizing outof-plane scattering, the present CR structure simultaneously achieves low insertion loss and high electrooptic spectral shift in a structure 50 times smaller in size compared to MZI devices, thus enabling fabrication of ultracompact electrooptic devices. With the combination of these attractive features, this new CRPC design is a promising structure for building electrooptic devices such as electric field sensors.

\section{Disclaimer}

The views and conclusions contained in this paper are those of the authors and should not be interpreted as representing the official policies, either expressly or implied, of the Defense Advanced Research Projects Agency or the U.S. Government.

\section{Conflict of Interests}

The authors declare that there is no conflict of interests regarding the publication of this paper.

\section{Acknowledgments}

This research was carried out in part at the Center for Functional Nanomaterials, Brookhaven National Laboratory, which is supported by the U.S. Department of Energy, Office of Basic Energy Sciences, under Contract no. DEAC02-98CH10886. This work was supported by the Defense Advanced Research Projects Agency, Electric Field Detector (E-FED) Program, under Grant HR0011-10-C-0043.

\section{References}

[1] M. Roussey, M.-P. Bernal, N. Courjal, D. Van Labeke, F. I. Baida, and R. Salut, "Electro-optic effect exaltation on lithium niobate photonic crystals due to slow photons," Applied Physics Letters, vol. 89, no. 24, Article ID 241110, 2006.

[2] H. Altug and J. Vučković, "Experimental demonstration of the slow group velocity of light in two-dimensional coupled photonic crystal microcavity arrays," Applied Physics Letters, vol. 86, no. 11, Article ID 111102, 2005.

[3] E. L. Wooten, K. M. Kissa, A. Yi-Yan et al., "A review of lithium niobate modulators for fiber-optic communications systems," IEEE Journal on Selected Topics in Quantum Electronics, vol. 6, no. 1, pp. 69-82, 2000.

[4] F. Chen, "Photonic guiding structures in lithium niobate crystals produced by energetic ion beams," Journal of Applied Physics, vol. 106, no. 8, Article ID 081101, 2009.

[5] S. S. Sriram and S. A. Kingsley, "Sensitivity enhancements to photonic electric field sensor," in Proceedings of the SPIE Defense \& Security Symposium, Orlando, Fla, USA, April 2004.

[6] O. Yavuzcetin, B. Ozturk, D. Xiao, and S. Sridhar, "Conicity and depth effects on the optical transmission of lithium niobate photonic crystals patterned by focused ion beam," Optical Materials Express, vol. 1, no. 7, pp. 1262-1271, 2011.

[7] H. Hu, R. Ricken, W. Sohler, and R. B. Wehrspohn, "Lithium niobate ridge waveguides fabricated by wet etching," IEEE Photonics Technology Letters, vol. 19, no. 6, pp. 417-419, 2007.

[8] O. Yavuzcetin, H. P. Novikov, R. L. Dally et al., "Photonic crystal fabrication in lithium niobate via pattern transfer through wet and dry etched chromium mask," Journal of Applied Physics, vol. 112, no. 7, Article ID 074303, 2012.

[9] W. C. L. Hopman, F. Ay, W. Hu et al., "Focused ion beam scan routine, dwell time and dose optimizations for submicrometre period planar photonic crystal components and stamps in silicon," Nanotechnology, vol. 18, no. 19, Article ID 195305, 2007.

[10] G. W. Burr, S. Diziain, and M.-P. Bernal, “The impact of finitedepth cylindrical and conical holes in lithium niobate photonic crystals," Optics Express, vol. 16, no. 9, pp. 6302-6316, 2008.

[11] A. Suzuki, T. Iwamoto, A. Enokihara, H. Murata, and Y. Okamura, "Fabrication of Bragg gratings with deep grooves in $\mathrm{LiNbO}_{3}$ ridge optical waveguide," Microelectronic Engineering, vol. 85, no. 5-6, pp. 1417-1420, 2008. 
[12] L. Pierno, M. Dispenza, A. Secchi, A. Fiorello, and V. Foglietti, "A lithium niobate electro-optic tunable Bragg filter fabricated by electron beam lithography," Journal of Optics A: Pure and Applied Optics, vol. 10, no. 6, Article ID 064017, 2008.

[13] K. Ghoumid, R. Ferrière, B.-E. Benkelfat, S. Mekaoui, C. Benmouhoub, and T. Gharbi, "Technological implementation Fabry-Pérot cavity in Ti: $\mathrm{LiNbO}_{3}$ waveguide by FIB," IEEE Photonics Technology Letters, vol. 24, no. 4, pp. 231-233, 2012.

[14] A. Yariv, Y. Xu, R. K. Lee, and A. Scherer, "Coupled-resonator optical waveguide: a proposal and analysis," Optics Letters, vol. 24, no. 11, pp. 711-713, 1999.

[15] O. Yavuzcetin, N. R. Perry, S. T. Malley et al., "Fabrication and characterization of single mode annealed proton exchanged waveguides in -x-cut lithium niobate," Optical Materials, vol. 36, no. 2, pp. 372-375, 2013.

[16] A. F. Oskooi, D. Roundy, M. Ibanescu, P. Bermel, J. D. Joannopoulos, and S. G. Johnson, "MEEP: a flexible free-software package for electromagnetic simulations by the FDTD method," Computer Physics Communications, vol. 181, no. 3, pp. 687-702, 2010.

[17] A. Guarino, G. Poberaj, D. Rezzonico, R. Degl'Innocenti, and P. Günter, "Electro-optically tunable microring resonators in lithium niobate," Nature Photonics, vol. 1, no. 7, pp. 407-410, 2007.

[18] J. E. Toney, V. E. Stenger, P. Pontius et al., "Photonic crystal electro-optic devices in engineered thin film lithium niobate substrates," in Photonic Microdevices/Microstructures for Sensing IV, vol. 8376 of Proceedings of SPIE, Baltimore, Md, USA, April 2012. 

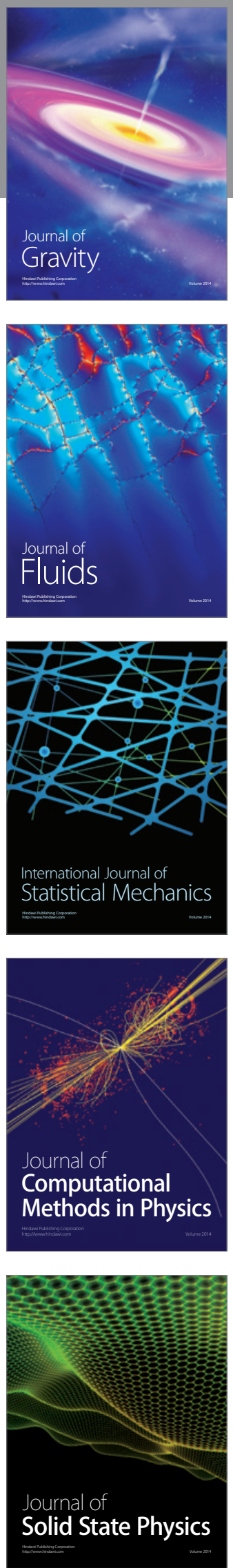

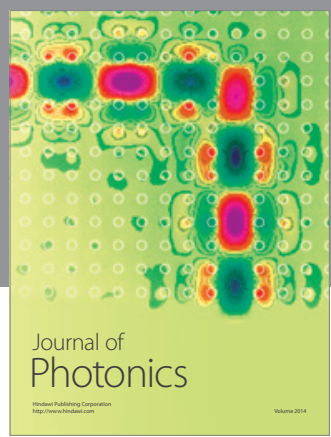

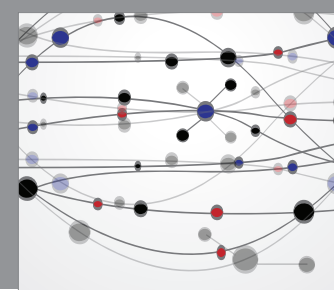

The Scientific World Journal

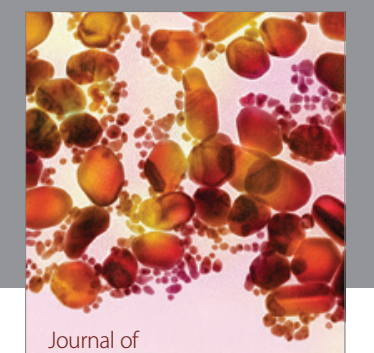

Soft Matter
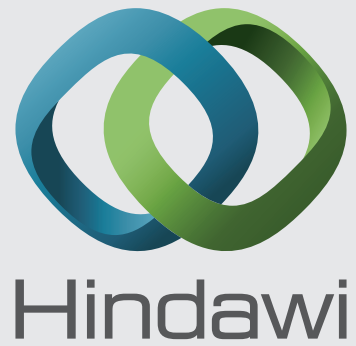

Submit your manuscripts at

http://www.hindawi.com
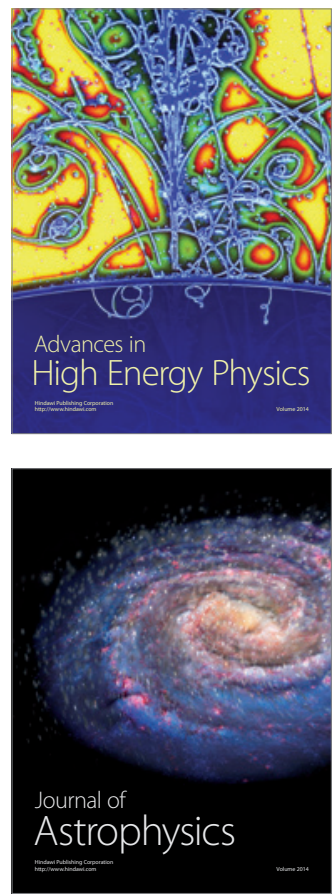
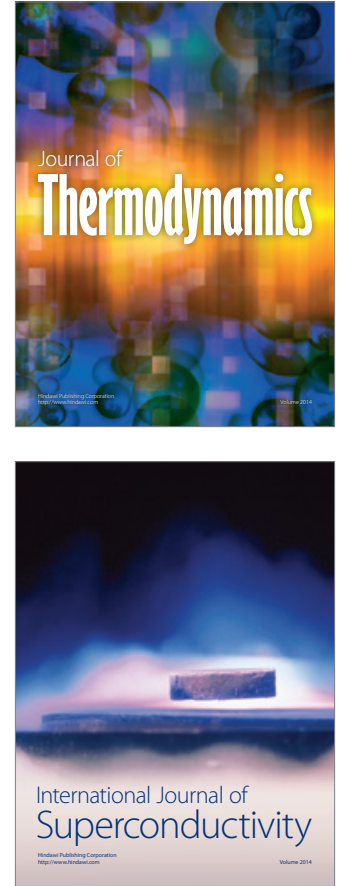
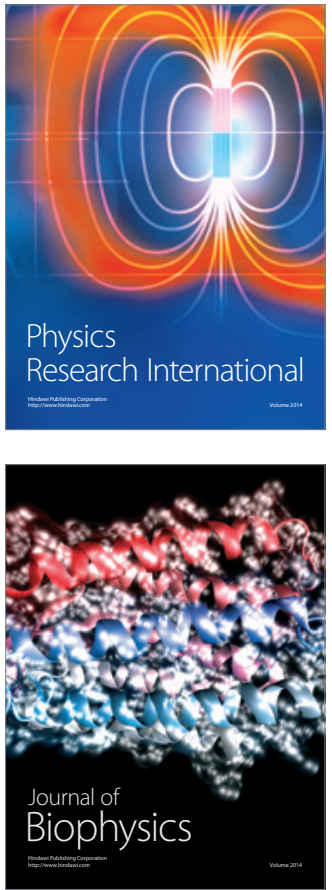
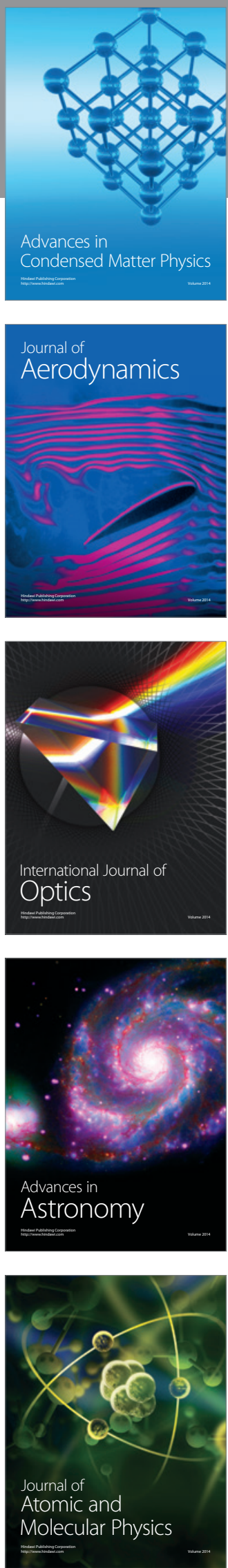